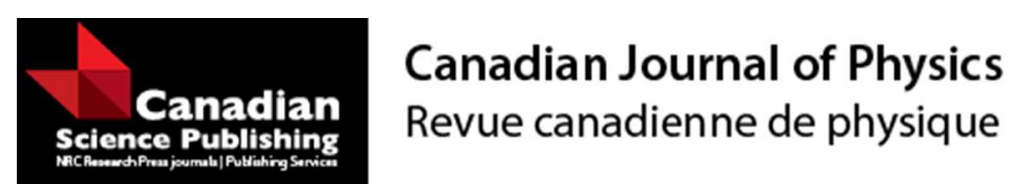

\title{
Vacuum Energy through Krein Quantization Approach
}

\begin{tabular}{|r|l|}
\hline Journal: & Canadian Journal of Physics \\
\hline Manuscript ID & cjp-2016-0013 \\
\hline Manuscript Type: & Article \\
\hline Date Submitted by the Author: & 06-Jan-2016 \\
\hline Complete List of Authors: & $\begin{array}{l}\text { Pirmoradian, Reza; Islamic Azad University Central Tehran Branch, Physics } \\
\text { Tavakoli, Farhad; Islamic Azad University Central Tehran Branch, Physics }\end{array}$ \\
\hline Keyword: & $\begin{array}{l}\text { cosmological constant, Renormalizing Process, zero-point energy, Vacuum } \\
\text { Energy, Krein Quantization }\end{array}$ \\
\hline
\end{tabular}

\section{SCHOLARONE ${ }^{\text {M }}$}

Manuscripts 


\title{
Vacuum Energy through Krein Quantization Approach
}

\author{
R. Pirmoradian and F. Tavakoli*
}

October 28, 2016

Department of Physics, Faculty of Science, Islamic Azad University Central Tehran Branch (IAUCTB), Tehran, Iran

\begin{abstract}
In this paper, we consider a new version of indefinite metric field quantization called "Krein" quantization approach. Centering on the vacuum energy, fundamental subjects revolve around this concept will be discussed. In this approach, vacuum expectation value of the energy-momentum tensor can be defined properly and uniquely. Actually, no infinite term appears and the vacuum energy of the free field vanishes. These properties allow us to propose a discussion which creates an interesting link to cosmological constant problem. Achieving this goal, however, necessitates consistency of the theory with conventional ones, so we have studied and made comparison essential issues such as unitarity of the theory, physical achievements of renormalizing process and the trace anomaly subject.
\end{abstract}

\section{Introduction}

The advent of divergence in expectation value of the energy-momentum tensor and consequently in zero-point energy leads to fundamental problems in modern physics. In non-gravitational physics, regarding this fact that only changes in energy from one state to another are measurable, any resulted infinite vacuum energy in QFT may be renormalized -or rescaled- by subtracting infinite energy from quantum vacuum energy [1, 2]. [An attractive force between two uncharged parallel conducting plates, which is known as Casimir force, showed that finite differences between different configurations of infinite energy do have physical reality [3].]

However, for some reasons, this simple device cannot be trusted in curved space-time, where the center equation for discussions of QFT's is the semi-classical Einstein's equation with a cosmological term, which creates a specific relation between the classical gravitational field and expectation value of the energy-momentum tensor $T_{\mu \nu}$,

$$
R_{\mu \nu}-\frac{1}{2} R g_{\mu \nu}+\Lambda g_{\mu \nu}=-8 \pi G<T_{\mu \nu}>
$$

where $R_{\mu \nu}$ and $R$ refer to the curvature of space-time, $g_{\mu \nu}$ to the metric, $\Lambda$ to the cosmological constant and $G$ to the Newton's constant.

First of all, in curved space-time, the gravitational field will in general be expected to produce particles, thereby there is no unique notion of a vacuum state or of particles $[1,4]$. So, though the choice of the Minkowskian vacuum defined by $\mid 0>$, has a well-understood physical meaning, i.e., the absence of particles according to all inertial observers, in general there is not any reference system in which there is no particle creation. With no clear vacuum concept, one cannot give a precise meaning to expectation value of the energymomentum tensor of the vacuum state, $<0\left|T_{\mu \nu}\right| 0>[1,4]$. Meanwhile, the source for the gravitational field is the entire energy-momentum tensor, which gives rise to space-time curvature. That means, the actual value of the energy matters, not just the differences between states. Therefore, the rescaling method cannot be expected to be valid generally.

On the other hand, recent astronomical observations indicate that the expansion of the universe is accelerating, which can be interpreted as an evidence for a non-vanishing positive cosmological constant [5] (or vacuum energy density). Without gravity, we do not care about the energy of the vacuum, but in the presence of gravity problems arise. According to the semi-classical Einstein's equation

$$
R_{\mu \nu}-\frac{1}{2} R g_{\mu \nu}+\Lambda g_{\mu \nu}=-8 \pi G<T_{\mu \nu}>
$$

*f _ tavakoli@iauctb.ac.ir 
( $R_{\mu \nu}$ and $R$ refer to the curvature of space-time, $g_{\mu \nu}$ to the metric, $\Lambda$ to the cosmological constant and $G$ to the Newton's constant), Lorentz invariance implies that in the vacuum, the energy-momentum tensor should be proportional to the metric, which proportionality coefficient is expectation value of the vacuum energy density

$$
\left\langle T_{\mu \nu}\right\rangle=\langle\rho\rangle g_{\mu \nu}
$$

(The metric chosen for flat space-time, has $g_{00}=+1$.) So, anything that contributes to the vacuum energy density, can be regarded as a cosmological constant

$$
\Lambda_{e f f}=\Lambda+8 \pi G\langle\rho\rangle
$$

If we can use QFT up to the Plank scale, we can calculate the upper bound for expectation value of the vacuum energy by discarding the very high momentum modes (cutoff method) as [6]

$$
\langle\rho\rangle \sim\left(10^{18} \mathrm{GeV}\right)^{4} \sim 10^{112} \mathrm{erg} / \mathrm{cm}^{3} .
$$

However, cosmological observations imply that the total effective vacuum energy, $\rho_{v}=\langle\rho\rangle+\frac{\Lambda}{8 \pi G}=\frac{\Lambda_{\text {eff }}}{8 \pi G}$, is $[7]$

$$
\rho_{v} \leq\left(10^{-12} \mathrm{GeV}\right)^{4} \sim 10^{-8} \mathrm{erg} / \mathrm{cm}^{3},
$$

which is much smaller than what we had expected from the calculations. This large discrepancy of $10^{120}$ order of magnitude is the world record on contradiction between the field theoretical calculations and the experimental results. Therefore, if the observations are correct, then $\rho_{v}$ is non-zero, very tiny and its value is extremely fine tuned for no good reason. This is a concrete statement of the first of the two cosmological constant problems [8].

Unfortunately as mentioned above, this is not the only problem related to cosmological constant. The second problem which we briefly state, is to understand why the vacuum energy is of the same order of magnitude as the present mass density of the universe [9].

In this work, we utilize "Krein" space quantization, a new version of indefinite metric field quantization, to peruse the above issues. We will show that some mentioned defects could be suppressed through this approach. The method is also capable of recovering the experimental results of the conventional QFT.

Krein space as the generalization of the Hilbert space is defined by $K=\mathcal{H}_{+} \oplus \mathcal{H}_{-}$, where $K$ is called the total space and $\mathcal{H}_{-}\left(=\mathcal{H}_{+}^{*}\right)$ stands for the anti-Hilbert space. Krein space quantization then is a canonical quantization of Gupta-Bleuler type in which the Fock space is constructed over the total space [10, 11, 12]. In this quantization method, the set of states is different from the set of physical states; The observables are defined on the total space, while the average values of the observables are calculated on the sub-space of physical states. It is worth to mention that the total space is equipped with an indefinite inner product which results in some (un-physical) states have a negative norm. An interesting feature of the theory is that, instead of having a multiplicity of vacuum, we have several possibilities for the space of physical states and only one field and one vacuum which are independent of Bogoliubov transformations [12]. So, the usual ambiguity about vacuum is not suppressed but displaced. This method, for the first time, was applied to the massless minimally coupled scalar field in de Sitter (dS) space $[13,14] .^{1}$ Furthermore, pursuing this path, it was shown that covariant quantization for massless spin-2 field in $\mathrm{dS}$ space is accessible [16, 17, 18, 19, 20].

Respecting the intrinsic specifications of Krein quantization method, vacuum expectation value of the energymomentum tensor is thoroughly discussed in this paper: Sec. (2) is devoted to a comprehensive introduction of Krein method, supplemented by new practical comments. Also, unitarity of the theory when interactions are taken into account and compatibility of the method with physical achievements of renormalizing process will be discussed in this section. In Sec. (3), the viewpoint of Krein method on the vacuum energy is perused. We show that the entrance of un-physical states in the theory, which act as intrinsic renormalizing devices, makes it possible to determine absolute meaning for energy. Then we consider the theory in the presence of boundary conditions, such as Casimir effect, and also in non-trivial topologies. Eventually, we study the results of generalizing the method to curved space-times, and we speak of Krein method faced with the trace anomaly subject and back reaction effect. Finally, in Sec. (4), respecting the Krein properties, we discuss about the cosmological constant problem; the method presents an interesting property linked to the cosmological constant problem "the vacuum energy of the free field vanishes without any reordering nor regularization".

\footnotetext{
${ }^{1}$ According to Allen's theorem [15], no invariant vacuum exists, therefore no covariant Hilbert space quantization is possible.
} 


\section{Krein Quantization}

\subsection{Basic Set-Up}

Let us illustrate Krein quantization procedure by giving a simple example, a Klein-Gordon-field in a twodimensional Minkowski space-time

$$
\left(\frac{\partial^{2}}{\partial t^{2}}-\frac{\partial^{2}}{\partial x^{2}}+m^{2}\right) \varphi(t, x)=0
$$

which has two sets of solutions

$$
\phi_{P}(k, t, x)=\frac{e^{i k x-i w t}}{\sqrt{(2 \pi) 2 w}}, \text { and } \phi_{N}(k, t, x)=\frac{e^{-i k x+i w t}}{\sqrt{(2 \pi) 2 w}},
$$

$w(k)=\left(k^{2}+m^{2}\right)^{\frac{1}{2}} \geq 0$. The inner product is defined as

$$
\left(\varphi_{1}, \varphi_{2}\right)=i \int_{t=\text { const }} d x\left(\varphi_{1}^{*} \partial_{t} \varphi_{2}-\varphi_{2} \partial_{t} \varphi_{1}^{*}\right)
$$

and these modes are normalized by the following relations

$$
\begin{gathered}
\left(\phi_{P}(k, t, x), \phi_{P}\left(k^{\prime}, t, x\right)\right)=\delta\left(k-k^{\prime}\right), \quad\left(\phi_{N}(k, t, x), \phi_{N}\left(k^{\prime}, t, x\right)\right)=-\delta\left(k-k^{\prime}\right), \\
\left(\phi_{P}(k, t, x), \phi_{N}\left(k^{\prime}, t, x\right)\right)=0 .
\end{gathered}
$$

The subscripts $P$ and $N$ are respectively referred to the positive and negative norm modes, or simply physical and un-physical states.

The field operator in Krein space is defined by

$$
\varphi(t, x)=\frac{1}{\sqrt{2}}\left(\varphi_{P}(t, x)+\varphi_{N}(t, x)\right)
$$

in which

$$
\begin{aligned}
& \varphi_{P}(t, x)=\int d k\left[a(k) \phi_{P}(k, t, x)+a^{\dagger}(k) \phi_{P}^{*}(k, t, x)\right], \\
& \varphi_{N}(t, x)=\int d k\left[b(k) \phi_{N}(k, t, x)+b^{\dagger}(k) \phi_{N}^{*}(k, t, x)\right] .
\end{aligned}
$$

The quantum field theory is identified by defining the vacuum states as

$$
\begin{aligned}
& a(k)|0\rangle=0, \quad a^{\dagger}(k)|0\rangle=\left|1_{k}\right\rangle, \\
& b(k)|0\rangle=0, \quad b^{\dagger}(k)|0\rangle=\left|\overline{1_{k}}\right\rangle,
\end{aligned}
$$

where $\left|\overline{1_{k}}\right\rangle$ is an un-physical state. A significant difference to the standard QFT, which is based on canonical commutation relation, lies in the requirement of the following commutation relations

$$
\begin{aligned}
& {\left[a(k), a^{\dagger}\left(k^{\prime}\right)\right]=\delta\left(k-k^{\prime}\right), \quad\left[a(k), a\left(k^{\prime}\right)\right]=\left[a^{\dagger}(k), a^{\dagger}\left(k^{\prime}\right)\right]=0,} \\
& {\left[b(k), b^{\dagger}\left(k^{\prime}\right)\right]=-\delta\left(k-k^{\prime}\right), \quad\left[b(k), b\left(k^{\prime}\right)\right]=\left[b^{\dagger}(k), b^{\dagger}\left(\overrightarrow{k^{\prime}}\right)\right]=0,} \\
& {[a(k), b(k)]=\left[a(k), b^{\dagger}(k)\right]=\left[a^{\dagger}(k), b(k)\right]=\left[a^{\dagger}(k), b^{\dagger}(k)\right]=0 .}
\end{aligned}
$$

\subsection{Unitarity of the Theory and Compatibility with Renormalizing Process}

In the context of Krein method, un-physical states are present. However, unitarity of the theory would be preserved by imposing the following conditions on quantum states and probability amplitude

- Un-physical states do not interact with the physical ones or real physical world. Indeed, in the Feynman diagrams, such states only appear in the internal legs and disconnected parts of the diagrams. 
- Un-physical states, which appear in the disconnected parts of the S-matrix elements, can be eliminated by renormalizing the probability amplitude as $[21,22]$

$$
S_{\text {if }}=\frac{\langle\text { physical states, in }| \text { physical states }, \text { out }\rangle}{\langle 0, \text { in }| 0, \text { out }\rangle} .
$$

The presence of un-physical states in the internal lines plays a key role in the renormalizing procedure, so that, by taking into account the quantum metric fluctuations, the divergences of the Green's function in QFT do not appear any more [23].

Considering these specifications, by using Krein approach, many topics have been studied and the results are in complete agreement with their (Hilbert space) QFT's counterparts, and experimental data. For instance, in [24] the one-loop effective action of QED is calculated by the Schwinger method in Krein approach. It is shown that the theory is free of any divergence and the effective action coincides with standard solution. The magnetic anomaly and lamb shift are also calculated in the one-loop approximation [21]. The results are comparable to the results of conventional QED. In Refs. [25, 26, 27, 28] the interaction QFT in Minkowski space $\left(\lambda \varphi^{4}\right.$ theory) is considered to the one-loop approximation. It is found that the theory is automatically renormalized in this approximation. In Refs. [29, 30] Casimir force in Minkowski space-time has been calculated. Once again it is indicated that the theory is automatically renormalized and the results are the same as those that have been experienced.

It should be noted that, the method can also reproduce the very results that are extracted from the renormalization group. To see the points of Krein method, we concentrate on a simple toy model, $\lambda \varphi^{4}$ scalar field, which is sufficient to give us the basic qualitative results of the approach. For this example, one can extract the effective potential in Krein view as follows [27]

$$
V_{e f f}=V_{e f f}^{(0)}+\hbar V_{e f f}^{(1)}+\hbar^{2} V_{e f f}^{(2)}+\ldots
$$

where

$$
\begin{gathered}
V_{\text {eff }}^{(0)}=\frac{m^{2} \varphi^{2}}{2}+\frac{\lambda \varphi^{4}}{4 !}, \\
V_{e f f}^{(1)}=-\frac{m^{2} \lambda \varphi^{2}}{(16 \pi)^{2}}\left[\left(1+\frac{\lambda \varphi^{2}}{m^{2}}\right)\left(-2 \ln \left(1+\frac{\lambda \varphi^{2}}{m^{2}}\right)+\ln \left(1+\frac{\lambda \varphi^{2}}{2 m^{2}}\right)+\ln \frac{\lambda \varphi^{2}}{2 m^{2}}+2 \ln 2\right)\right. \\
\left.+\ln \left(1+\frac{\lambda \varphi^{2}}{2 m^{2}}\right)-\ln \frac{\lambda \varphi^{2}}{2 m^{2}}\right]-\frac{m^{2} \lambda \varphi^{2}}{64 \pi}\left[1+\sqrt{1+\frac{2 m^{2}}{\lambda \varphi^{2}}}-2 \sqrt{1+\frac{m^{2}}{\lambda \varphi^{2}}}\right] .
\end{gathered}
$$

As expected, classically, one can acquire the coupling constant by four times differentiating of the $V_{e f f}^{(0)}$ with respect to $\varphi$. The result would be obtained to order $\hbar^{0}$ as shown by (12). Following the same path, in order to include the quantum corrections in the theory, we accomplish four times differentiating of the $V_{e f f}$ with respect to $\varphi$ and then, substitute $\varphi$ with a non-zero arbitrary parameter $M$. The result will be as [27]

$$
\lambda^{\text {Krein }}=\lambda-\frac{\lambda^{2}}{(8 \pi)^{2}}\left[6 \ln \frac{M^{2}}{m^{2}}+19+12 \ln 2\right]+\mathcal{O}\left(\lambda^{3}\right) .
$$

Like routine methods, the dependence of the coupling constant, $\lambda^{\text {Krein }}$, on the mass scale, $M$, is governed by a beta function of the theory, which is defined as

$$
\beta^{\text {Krein }}=M \frac{\partial \lambda^{\text {Krein }}}{\partial M},
$$

so that, by applying this process to $\lambda \varphi^{4}$ scalar field theory, one can obtain [27]

$$
\beta^{\text {Krein }}=\frac{3 \lambda^{2}}{16 \pi^{2}},
$$

which only depends on coupling constant and has no explicit dependence on $M$.

It is worth mentioning that, although the effective potential and coupling constant calculated by using Krein procedure are different from those that come from conventional procedure, however, both methods yield the same beta function. This process can be applied to the other QFTs to calculate the beta function as it has been carried out for QED [31]. Concentrating on the beta function this much is due to the fact that it has important physical outcomes, such as the trace anomaly subject which would be discussed in Sec. (4). 


\section{Vacuum Energy in Krein Quantization Approach}

In physics there is a great variety of viewpoints of what vacuum energy is. It is understood differently in many formalisms and domains of study. In the field theory approach, a quantum field can be regarded as a collection of an infinite number of harmonic oscillators in momentum space, therefore, expectation value of the energy-momentum tensor and as a result zero-point energy of such an infinite collection will be infinite. Various ways have been contrived to deal with this infinite overall energy. In what follows, we study Krein quantization approach, as an alternative method, to address this problem and show how in this method, expectation value of the energy-momentum tensor of the vacuum state can be defined properly and uniquely.

\subsection{Applying the Method in Flat Space-times}

At this stage, we wish to restrict attention to space-times where we are able to formulate quantum field theory constructions in a manner completely analogous to Minkowski space-time. In a two-dimensional Minkowski space-time, the energy density operator is given by the 00-component of the energy-momentum tensor

$$
T_{00}=\frac{1}{2}\left\{\left[\partial_{t} \varphi(t, x)\right]^{2}+\left[\partial_{x} \varphi(t, x)\right]^{2}\right\}
$$

Regarding the Krein technique, the field operator is constructed by both physical and un-physical states, so unlike the conventional methods which rescale energy to be released from infinities, vacuum energy in Minkowski space-time automatically vanishes, i.e. $\left\langle 0\left|T_{00}^{\mathrm{Krein}}\right| 0\right\rangle=0,|0\rangle$ is the Minkowski space vacuum. In fact, by utilizing un-physical states as renormalizing tools, energy itself is meaningful and can be defined absolutely. While, in standard process, the vanishing vacuum expectation value of the energy in Minkowski space-time is respected as the physical "renormalization condition" [2].

Now we will take the field operator (7) to calculate vacuum energy in the presence of boundary conditions to test the method in a physical measurable case and then we make comparison with the standard approach. It is preferred that the boundary conditions being separated into two categories: physical and intrinsic boundary conditions. By physical boundary conditions, contrary to the intrinsic boundary conditions (as the periodic boundary condition on $R^{1} \times S^{1}$ space-time), we mean the conditions, that can be demonstrated by a potential term in the Lagrangian of the system. At the first step, we examine vacuum energy between two parallel conducting plates as the simplest case of physical boundary conditions. By imposing the physical boundary condition on the field operator (7), only physical states would be affected and un-physical states do not interact with the physical ones or real physical world. Therefore, the field operator can be written as:

$$
\varphi(t, x)=\sum_{d}\left[a\left(k_{d}\right) \phi_{P}\left(k_{d}, t, x\right)+a^{\dagger}\left(k_{d}\right) \phi_{P}^{*}\left(k_{d}, t, x\right)\right]+\int d k\left[b(k) \phi_{N}(k, t, x)+b^{\dagger}(k) \phi_{N}^{*}(k, t, x)\right],
$$

here $k_{d}$ are the eigen-frequencies of the system under consideration, where in this case; $\phi_{P}(k, t, 0)=\phi_{P}(k, t, a)=$ 0 , we have

$$
\phi_{P}\left(k_{d}, t, x\right)=\left(\frac{1}{a \omega_{d}}\right)^{1 / 2} e^{-i \omega_{d} t} \sin k_{d} x
$$

where $\omega_{d}=\left(m^{2}+k_{d}^{2}\right)^{1 / 2}$ and $k_{d}=\frac{d \pi}{a}, d=1,2,3, \ldots$ The scalar product is:

$$
\left(\phi_{P}(k, t, x), \phi_{P}\left(k^{\prime}, t, x\right)\right)=\delta_{k k^{\prime}}
$$

Substituting field operator (17) into (16) leads to:

$$
<0\left|T_{00}^{\mathrm{Krein}}\right| 0>=\frac{1}{2 a} \sum_{d=1}^{\infty} \omega_{d}-\frac{m^{2}}{2 a} \sum_{d=1}^{\infty} \frac{\cos 2 k_{d} x}{\omega_{d}}-\frac{1}{2 \pi} \int_{0}^{\infty} \omega(k) d k
$$

Then total vacuum energy of the interval $(0, a)$ is obtained as follow [29]:

$$
E=\int_{0}^{a}<0\left|T_{00}^{\mathrm{Krein}}\right| 0>d x=-\frac{\pi}{24 a} .
$$


Surveying the Eq. (18) reveals that in standard method with the choice of Hilbert space as the space of positive frequency solutions, only the first two terms are apparent, which cause an infinite result. In order to remove this infinity or renormalize the theory, one rescales the energy by subtracting the Minkowski vacuum energy, so the third term is added to the equation manually. However, in Krein method, because of a new interpretation of un-physical states, as natural renormalizing tools, the third term will appear spontaneously. Therefore, an absolute meaning for energy is achieved and consequently the result is automatically renormalized and exactly coincides with conventional method and the measured value [3].

Now we study Krein method in non-trivial topologies. As a toy model, we choosed a two-dimensional flat cylindrical space-time $\left(R^{1} \times S^{1}\right)$ with periodicity length, $L$. Although this topology is not physical and so no measurements can be made, but studying this model is instructive, cause it has some similarities to dS curved space-time $\left(R^{1} \times S^{3}\right)$ which has similar intrinsic boundary condition. For a massless Klein-Gordon-field we have:

$$
\frac{\partial^{2} \varphi}{\partial t^{2}}-\frac{\partial^{2} \varphi}{\partial x^{2}}=0
$$

In this case, the effect of the space closure (intrinsic boundary condition), contrary to the physical ones, restricts the both positive and negative energy states. Since according to the fundamental postulates of QFT, the spinless wave function must have a definite value at every point in space [32]. Hence, we must demand that the field modes (4) be single-valued, and so, the field operator (7) converts to

$$
\varphi(t, x)=\sum_{n=-\infty}^{\infty}\left[a\left(k_{n}\right) \phi_{P}\left(k_{n}, t, x\right)+a^{\dagger}\left(k_{n}\right) \phi_{P}^{*}\left(k_{n}, t, x\right)\right]+\sum_{n=-\infty}^{\infty}\left[b\left(k_{n}\right) \phi_{N}\left(k_{n}, t, x\right)+b^{\dagger}\left(k_{n}\right) \phi_{N}^{*}\left(k_{n}, t, x\right)\right],
$$

in which

$$
\phi_{P}=\frac{e^{i\left(k_{n} x-\omega t\right)}}{\sqrt{2 L \omega}}, \text { and } \phi_{N}=\frac{e^{i\left(-k_{n} x+\omega t\right)}}{\sqrt{2 L \omega}},
$$

with $k_{n}=\frac{2 n \pi}{L}, n=0, \pm 1, \pm 2, \ldots$ and $\omega=|k|$. Vacuum energy density of such field on $R^{1} \times S^{1}$ can be calculated as follows

$$
\left\langle 0_{L}\left|T_{00}^{\mathrm{Krein}}\right| 0_{L}\right\rangle=\frac{2 \pi}{L^{2}} \sum_{n=0}^{\infty} n-\frac{2 \pi}{L^{2}} \sum_{n=0}^{\infty} n=0 .
$$

Therefore the vacuum energy is automatically renormalized and it is equal to zero. As before in standard method, just the first term of (22) exists, which is obviously infinite. This was expected, as the $R^{1} \times S^{1}$ spacetime suffers from the same ultraviolet divergence properties as Minkowski space [2], thus the renormalizing process will be accomplished by subtracting the infinite quantum vacuum energy of Minkowski space-time. Therefore, we have [33]

$$
\left\langle 0_{L}\left|: T_{00}:\right| 0_{L}\right\rangle=\left\langle 0_{L}\left|T_{00}\right| 0_{L}\right\rangle-\left\langle 0\left|T_{00}\right| 0\right\rangle=\frac{2 \pi}{L^{2}} \sum_{n=0}^{\infty} n-\int_{0}^{\infty} k d k=-\frac{\pi}{6 L^{2}},
$$

where the symbol ": :" refers to the energy rescaling process and $\left|0_{L}\right\rangle,|0\rangle$ are the vacuum associated with the discrete modes (21) and the Minkowski space, respectively. So, there is a quite different result.

Thus far, we have studied non-gravitational cases in Krein quantization method. The main result obtained is that, due to its new interpretation of un-physical states, namely playing a renormalizing role, an absolute meaning for energy can be defined. By using Krein procedure, remarkable results have been achieved: Vacuum expectation value of the energy-momentum tensor automatically vanishes with no need to rescaling energy. In addition, in the presence of the physical boundary conditions, which lead to non-uniform distribution of energy density, ${ }^{2}$ even though the both methods, standard and Krein methods, are in agreement with experience (at least in Minkowski space-time [29, 30]), but the perspective and procedure of these two methods are different. In the standard method, the finite results are acquired by a process named renormalization. In the Krein method, infinities are eliminated and theories are automatically renormalized. However, in non-trivial topologies, in the absence of any experimental evidence, the disagreement between two methods appears, which shows a path that may be some help in solving problems in curved space-times.

\footnotetext{
${ }^{2}$ Actually, the difference of energy densities between parallel plates and outer regions, has been calculated as Casimir force.
} 


\subsection{Generalizing the Method to Curved Space-times}

An alternative strategy to inspect the divergence of expectation value of the energy-momentum tensor in curved space-time, regarding the semi-classical approach, is to treat the computation of $\left\langle T_{\mu \nu}\right\rangle$ as a part of a wider dynamical theory involving gravity; Such semi-classical field equation, (2), of a given coupled gravitationalquantum matter field, can be derived from the following action

$$
S=S_{g}+W
$$

by the condition $\left(g \equiv\left|\operatorname{det} g_{\mu \nu}\right|\right)$

$$
\frac{2}{\sqrt{-g}} \frac{\delta S}{\delta g^{\mu \nu}}=0
$$

$S_{g}$ is the gravitational action for which $2(-g)^{-\frac{1}{2}} \delta S_{g} / \delta g^{\mu \nu}$ yields the left hand side of (2). The second term in $(24)$ is the effective action of the quantum matter fields, which, when functionally differentiated, leads to $<T_{\mu \nu}>$, i.e.

$$
\frac{2}{\sqrt{-g}} \frac{\delta W}{\delta g^{\mu \nu}}=<T_{\mu \nu}>
$$

In this perspective, computation of the effective action has some interesting and non-trivial consequences, amongst them, the trace anomaly is the most important one for this paper. The trace (conformal) anomaly is a quantum anomaly of the semi-classical gravitation that not only has been connected with but also has influenced many important issues in modern physics. A good approach to a more detailed discussion would be an investigation of a self-interacting scalar field, $\lambda \varphi^{4}$ theory, with the following conformal invariant classical matter action in curved space-time

$$
S_{m}=\int \mathcal{L} d^{4} x
$$

where

$$
\mathcal{L}=\mathcal{L}_{0}+\mathcal{L}_{I}=\frac{1}{2} \sqrt{-g}\left(g^{\mu \nu} \partial_{\mu} \varphi \partial_{\nu} \varphi-\frac{1}{6} R \varphi^{2}\right)-\frac{1}{4 !} \sqrt{-g} \lambda \varphi^{4},
$$

the subscripts 0 and $I$ are respectively referred to free field and interaction-dependent parts of the theory. Classically, conformal invariant theories in which the matter action is invariant under conformal transformations $\left(g_{\mu \nu}(x) \rightarrow \Omega^{2} g_{\mu \nu}(x)=\bar{g}_{\mu \nu}(x)\right)$, yield a zero trace of the classical energy-momentum tensor [1]. When quantum corrections are included, however, this classical invariance cannot be preserved, and gives rise to an anomalous trace. This quantum anomaly in Krein quantization approach, like standard method [1], can be separated into two parts, cause the effective action can be written as

$$
W^{\text {Krein }}=W_{0}^{\text {Krein }}+W_{I}^{\text {Krein }},
$$

where $W_{0}^{K r e i n}$ is the free field effective action and $W_{I}^{K r e i n}$ is the interaction-dependent part which have been calculated in Krein quantization method. Then, the trace of expectation value of the energy-momentum tensor would be (by using $\delta \bar{g}^{\mu \nu}(x)=-2 \bar{g}^{\mu \nu}(x) \Omega^{-1}(x) \delta \Omega(x)$.)

$$
<T_{\mu}^{\mu}>^{\text {Krein }}=-\left.\frac{\Omega(x)}{\sqrt{-g(x)}} \frac{\delta W^{K r e i n}\left[\bar{g}_{\mu \nu}\right]}{\delta \Omega(x)}\right|_{\Omega=1}=<T_{\mu}^{\mu}>_{0}^{\text {Krein }}+<T_{\mu}^{\mu}>_{I}^{K r e i n}
$$

The first term on the right is the manifestation of the gravitational background which corresponds to the calculation of the free field part of the theory. As already stated, in Krein technique, influenced by un-physical states, the free field part of vacuum expectation value of the energy-momentum tensor automatically vanishes, and so, its trace will be removed. While in the standard approach, confronting the divergence of $\left\langle T_{\mu}^{\mu}\right\rangle_{0}$ is inevitable, where, by pursuing a careful renormalizing procedure, free field effective action can be divided into divergent and renormalized parts, i.e. $W_{0}=\left(W_{0}\right)_{r e n}+\left(W_{0}\right)_{\text {div }}$, in which $\left(W_{0}\right)_{\text {div }}$ is purely geometrical, built out of the Riemann curvature tensor, $R_{\mu \nu \rho \tau}$, and its contractions [1]. So, according to Eq. (26), one can split the right hand side of the semi-classical Einstein's equation (2) into two parts, purely geometrical part and dependent part on quantum states of the matter fields. On the other hand, the conformal invariance of the total action (27) requires that the total energy-momentum tensor should be traceless

$$
<T_{\mu}^{\mu}>_{0}=\left(<T_{\mu}^{\mu}>_{0}\right)_{d i v}+\left(<T_{\mu}^{\mu}>_{0}\right)_{r e n}=0,
$$


in which the non-vanishing value of $\left(<T_{\mu}^{\mu}>_{0}\right)_{d i v}$, the geometrical part, compels the renormalized energymomentum tensor to possess a trace [1]. This result is known as the trace anomaly of the free field part of the theory, which is state-independent and local. As mentioned, this part does not appear in the viewpoint of Krein method.

However, the second term in Eq. (30), which is due to the interaction-dependent part of the theory, remains. Actually, affected by the unavoidable presence of the arbitrary mass scale (see Eq. (14) and the related discussions), the conformal transformation of the theory leads to a different value of the effective coupling constant in Krein viewpoint, $\lambda^{\text {Krein }}$, even if the corresponding classical theory is conformal invariant. Therefore, in Krein's point of view, the trace anomaly is just limited to the interaction-dependent part of the theory, which contrary to the free field trace anomaly, is state-dependent and non-local

$$
<T_{\mu}^{\mu}>^{\text {Krein }}=<T_{\mu}^{\mu}>_{I}^{\text {Krein }}=\frac{1}{\sqrt{-g}}\left(\left(\beta^{\text {Krein }}\right) \frac{\delta}{\delta\left(\lambda^{\text {Krein }}\right)}\right)\left(W_{I}^{\text {Krein }}\right) .
$$

Obviously, the interaction-dependent anomalous breaking of conformal invariance is directly related to the beta function of the theory. It is worth to mention that, this interaction-dependent trace anomaly, as the common point of view between Krein method and the other standard approaches, ${ }^{3}$ in the chiral limit of quantum chromodynamics (QCD), determines the sizes and masses of hadrons, including but not limited to protons and neutrons [37, 38].

Here, we should underline that, removing the free field part of expectation value of the energy-momentum tensor through the Krein method leads to the loss of geometrical effects, caused by renormalizing procedure in the standard approach, on the semi-classical Einstein's equation (see Ref. [39] and also the related discussion in the previous section). While, in the conventional viewpoint, accepting the influence of the renormalizing process of expectation value of the energy-momentum tensor on geometrical part of Einstein's equation, represents a "back-reaction" effect which needs to be dealt with in some self-consistent ways. If $\left\langle T_{\mu \nu}>\right.$ affects the metric, then this altered metric will change the assumptions for calculating $\left\langle T_{\mu \nu}\right\rangle$. Technically, it is extremely difficult to calculate $\left\langle T_{\mu \nu}\right\rangle$ in a given fixed background and incorporation of the back-reaction effect makes it close to impossible (for a more detailed discussion, one can refer to Ref. [40]).

\section{Discussion}

It is well-known that cosmological constant is directly related to the vacuum energy density or more precisely vacuum expectation value of the energy-momentum tensor. As pointed out, when gravity is present, the very existence of cosmological constant brings about fundamental problems. However, respecting the fact that many great progressions in physics are done in crises, various attempts have been made in trying to solve the cosmological constant problem. Indeed, understanding why the cosmological constant is extremely small may be a key for making more realistic models of particle physics and/or gravitation. Some of the main approaches to the problem can be listed as: Supersymmetry, Brane-World scenarios, String Theory inspired ideas, Quintessence, Anthropic interpretation of the cosmological constant and so forth (see, for instance, [8, 36] and references therein). But up to now, a convincing and general solution to the cosmological constant problem has not been yet proposed. Definitely, a fundamental theory of the universe is required to give a complete apprehension of the problem and probably a plausible solution for it. Science such an elemental theory is unknown, any effective description can be considerable, although incomplete.

Following this path, we prove that by using Krein space quantization approach, one can propose a solution to the huge discrepancy between theoretical computation and cosmological data. According to explanations stated in the introduction and the expression for the total vacuum energy, $\rho_{v}=\langle\rho\rangle+\frac{\Lambda}{8 \pi G}=\frac{\Lambda_{\text {eff }}}{8 \pi G}$, the energy density of the vacuum, $\langle\rho\rangle$, is responsible for the immense discrepancy between theoretical predictions and experimental observations. However in Krein technique, vacuum expectation value of the energy-momentum tensor which is the very vacuum energy density, globally ${ }^{4}$ vanishes. As a result, the "vacuum energy density" term, $\langle\rho\rangle$, which creates such enormous disagreement between theory and experiment is automatically eliminated. Nevertheless, the authors would like to mention that this approach cannot be considered as a perfect

\footnotetext{
${ }^{3}$ As stated in Sec. (2), at least, the Krein beta functions of the theories that have been studied till today, coincide with the ones that had been obtained in standard approach [27, 31].

${ }^{4}$ As proved, vacuum expectation value of the energy-momentum tensor is independent of the curvature.
} 
solution to the whole cosmological constant problem, but it can be enlightening and provide some arguments for its possible elaboration in modern physics.

Acknowledgements

We would like to thank H. Pejhan and R. Dehghan for their early corporation in this work. This work was supported by a grant from Islamic Azad University, Central Tehran Branch.

\section{References}

[1] N.D. Birrell, P.C.W. Davies, Cambridge University Press, (1982), Quantum Field in Curved Space.

[2] B.S. Kay, Phys. Rev. D 20, 3052 (1979).

[3] H.B.G. Casimir, Proc. K. Ned. Akad. Wet. 51, 793 (1948).

[4] S.E. Rugh and H. Zinkernagel, Studies in History and Philosophy of Modern Physics, 33, 663-705 (2002).

[5] A. Balbi et al. ApJ 545, 1 (2000), Constraints on Cosmological Parameters from MAXIMA-1.

[6] S. Weinberg, Rev. Mod. Phys. 61, 1 (1989);

S.M. Carroll, Liv. Rev. Rel 4, 1 (2001).

[7] A.G. Riess et al. (Supernova Search Team Collaboration), Astrophys. J. 116, 1009 (1998);

A.G. Riess et al., Astrophys. J. 98, 659 (2007).

[8] T. Padmanabhan, Physics Reports 380, 5-6 (2003).

[9] S. Weinberg, The Cosmological Constant Problems, arXiv:astro-ph/0005265.

[10] M. Mintchev, J. Phys. A 13, 1841-1860 (1990).

[11] J. Bognar, Springer-Verlag, Berlin (1974), Indefinite inner product space.

[12] T. Garidi, E. Huguet and J. Renaud, J. Phys. A 38, 245 (2005).

[13] J.P. Gazeau, J. Renaud, and M.V. Takook, Class Quant. Grav. 17, 1415 (2000).

[14] S. De Bievre, J. Renaud, Phys. Rev. D 57, 6230 (1998).

[15] B. Allen, Phys. Rev. D 32, 3136 (1985);

B. Allen, A. Folacci, Phys. Rev. D 35, 3771 (1987).

[16] M.V. Takook, Mod. Phys. Lett. A. 16, 1691 (2001).

[17] S. Behroozi, S. Rouhani, M.V. Takook and M.R. Tanhayi, Phys. Rev. D 74, 124014 (2006).

[18] M. Dehghani, S. Rouhani, M.V. Takook and M.R. Tanhayi, Phys. Rev. D 77, 064028 (2008).

[19] T. Garidi, J.P. Gazeau, S. Rouhani and M.V. Takook, JMP 49, 032501 (2008).

[20] M.V. Takook and S. Rouhani, Quantum Linear Gravity in de Sitter Universe I: On Gupta-Bleuler vacuum state, arXiv:1208.5562.

[21] B. Forghan, M.V. Takook and A. Zarei, Annals of Physics 327, 2388 (2012).

[22] B. Forghan, M. R. Tanhayi, Mod. Phys. Lett. A 30 (26), 1550126 (2015).

[23] M.V. Takook, Int. J. Mod. Phy. E. 14219 (2005).

[24] A. Refaei, M.V. Takook, Phys. Lett. B 704, 326 (2011).

[25] M.V. Takook, Int. J. Mod. Phys. E 11, 509 (2002).

[26] S. Rouhani and M.V. Takook, Euro. Phys. lett. 68, 15 (2004). 
[27] A. Refaei, M.V. Takook, Mod. Phys. Lett. A. 26, 31 (2011).

[28] B. Forghan, Int. J. Theor. Phys. 51, 5 (2012).

[29] H. Khosravi, M. Naseri, S. Rouhani and M.V. Takook, Phys. Lett. B 640, 48 (2001).

[30] H. Pejhan, M.R. Tanhayi, M.V. Takook, Annals of Physics 341, 195 (2014).

[31] B. Forghan, Int. J. Theor. Phys. 51, 12 (2012).

[32] E. Merzbacher, Am. J. Phy., 30, 237 (1962).

[33] M. Bordag, U. Mohideen, V.M. Mostepanenko, Phys. Rept. 353, 1-205 (2001).

[34] S.A. Fulling, Aspects of Quantum Field Theory in Curved Spacetime, Cambridge University Press, Cambridge (1989).

[35] C.J. Isham, Quantum field theory in curved spacetimes: A general mathematical framework in Differential geometrical methods in mathematical physics II, Lecture Notes in Math., vol 676, ed. by K. Bleuler et alii, Springer, Berlin (1978).

[36] A. Vilenkin, Cosmological constant problems and their solutions, arXiv:hep-th/0106083.

[37] L.D. Rose, The Standard Model in a Weak Gravitational Background. arXiv:1308.0124.

[38] V.M. Sarti, Universit'a degli studi di Ferrara, (2012), Scaled chiral quark-solitons for nuclear matter.

[39] M.R Tanhayi and R. Pirmoradian, International Journal of Theoretical Physics, DOI 10.1007/s10773-0152714-z, (2015).

[40] R.M. Wald, (1994), Quantum Field Theory in Curved Space-time and Black Hole Thermodynamics. 